\title{
VARIOUS MAGNET OPTIONS FOR DELTA LAUNCHED VERSION OF ASTROMAG
}

\author{
Michael A. Green \\ Lawrence Berkeley Laboratory \\ University of California \\ Berkeley, CA 94720 \\ Cryogenic Engineering Conference \\ Albuquerque Conference Center \\ Albuquerque, New Mexico \\ 12-16 July 1993 \\ To be published in the \\ Advances in Cryogenic Engineering Vol 39
}

*This work was performed at the Lawrence Berkeley Laboratory with the support of the Office of Astrophysics, NASA and the Director of the Office of Energy Research, Office of High Energy and Nuclear Physics, High Energy Physics Division, U. S. Department of Energy under Contract No. DE-AC03-76SF00098.

\section{MASTER}




\title{
VARIOUS MAGNET OPTIONS FOR A DELTA LAUNCHED VERSION OF ASTROMAG
}

\author{
M. A. Green \\ Lawrence Berkeley Laboratory \\ University of California \\ Berkeley, CA 94720
}

\begin{abstract}
This Report compares various superconducting magnet options for use in a particle astrophysics experiment to be launched with a Delta rocket into a sun synchronous orbit and an earth trailing solar orbit. The goals of the experiment are to study the incidence of anti particles coming from deep space and to study populations of light nuclei (up to atomic number 8) and gammas from deep space. The proposed superconducting coils, which would be made with an aluminum stabilized niobium titanium superconductor, would be cooled using stored super fluid helium. Both a sun synchronous orbit and an earth trailing solar orbit permit one to shield the magnet and cryogen vessel behind the solar panels. This permits one to reduce the temperature of the cryostat outer vessel that increases to cryogen life time for the experiment. A number of superconducting coil and cryostat options were looked at in terms of total magnet and cryostat mass, cryogen life time, complexity and overall particle astrophysics capability for attached detectors.
\end{abstract}

\section{BACKGROUND}

ASTROMAG was originally conceived as an experiment for the space station 1.2 . The primary scientific goals of ASTROMAG were: 1) to test cosmological models by searching for antimatter and dark matter candidates, 2) to study the origin and evoluticn of matter in the galaxy by sampling the nuclei in cosmic rays originating in deep space and comparing the abundance of these nuclei with the solar system, and 3) to study the origin and acceleration of relativistic particles in the galaxy and their effects on the evolution of the galaxy.

The magnetic field for bending the charged particles from space will be provided by a short superconducting solenoid magnet. The magnet will consist of two nearly identical superconducting coils on the two ends of a super fluid helium storage tank ${ }^{2}$. The two coils will operate at opposite polarity so that the net magnetic dipole moment generated by the magnet system is close to zero. As a result, the magnet will not follow an earth's magnetic field line. (For the space station version of ASTROMAG, the two oppositely powered coils are good enough to prevent the superconducting magnet system from applying undue torque on the space station.) The ASTROMAG superconducting magnet system will operate at $1.8 \mathrm{~K}$, with cooling provided by super fluid helium stored in the tank between the two coils. Super fluid helium is kept in the storage tank by a porous plug phase separator ${ }^{3}$. 
An expendable launch vehicle configuration of ASTROMAG became desirable when the Space Station could no longer provide electric power and cooling to ASTROMAG. A decision was made to downsize ASTROMAG so that it could be launched by an Atlas IIa launch vehicle in a $500 \mathrm{~km}$ high, 57 degree circular orbit.5. The use of the Atlas IIa permitted one to la'inch a version of ASTROMAG that would have both the WiZard experiment (an anti particle cxperiment for measuring the spectra of electrons, positrons, anti-protons and other particles) and the LISA experiment (a heavy nuclei experiment for measuring the momentumı and energy of nuclei with an atomic number from 3 to 31). The choice of a 57 degree orbit is dictated by the Atlas launch facility at the Kennedy Space Flight Center. An advantage of the 57 degree is the increased flux of particles that have an energy of less than $1 \mathrm{GeV}$ per nucleon. The disadvantages of using an expendable launch vehicle such as the Atlas ila are: 1) The mass that can be put into orbit for ASTROMAG is limited to $5200 \mathrm{~kg}$ or less; 2) superconducting dipole moment correction coils ${ }^{6}$ are needed to keep the ASTROMAG from tracking the earth's magnetic field; 3 ) the satellite must supply all of the power, the telemetry and cooling for the experiment (About 25 percent of the launch mass is the satellite support system.); and 4) The mass of superconducting magnet and its cryostat has to be reduced from $2280 \mathrm{~kg}$ for the space station version to $1590 \mathrm{~kg}$ for the Atlas IIa version. By changing the matrix material in conductor in the ASTROMAG superconducting coil from a copper to very pure aluminum (RRR > 1000 at zero field), the number of ampere turns in the Atlas version can be increased enough to comperisate for the reduction of coil diameter ${ }^{7}$. (As a result, the particle resolution of the magnet was changed very little.)

A further downsizing of ASTROMAG has been dictated by the desire to use a launch vehicle which is less expensive to launch. A Delta II launch vehicle was selected as a candidate less expensive launch vehicle ${ }^{8}$. This vehicle can be launched from the west coast of the United States as well as the Kennedy Space Flight Center in Florida. The California launch facility permits one to launch ASTROMAG into a $800 \mathrm{~km}$ sun synchroncus circular orbit (a 98.7 degree polar orbit). Such an orbit permits the experiment to be powered by smaller solar panels and the number of low energy particles (less than $1 \mathrm{GeV}$ per nucleon) is increased over the 57 degree orbit. The amount of material that can be put into a sun synchronous orbit varies from $2570 \mathrm{~kg}$ to $3260 \mathrm{~kg}$ depending on the type of Delta II launch vehicle used. The maximum size Delta II fairing is 3.05 meters (10 feet) in diameter which means that the maximum dimensions of an ASTROMAG satellite is limited to 2.79 meters in diameter and 5.73 meters long. (If a $2.44 \mathrm{~m}$ ( $8 \mathrm{foot}$ ) fairing with a bulge is used, the maximum diameter in the bulge region is about 2.6 meters.) For ASTROMAG, the fairing diameter is probably not an issue. The real issue is the length available for the satellite and the ASTROMAG magnet and the two experimental packages. This limitation may require both ASTROMAG experiments to share the same end of the magnet. Even under the best scenario, only one large experiment can be accommodated. The mass of the of sun synchronous orbit Delta magnet version is about 1000 $\mathrm{kg}$. The reduced coil size is not compensated for by an increase in ampere turns. As a result, the particle resolution of the magnet is reduced. This reduction affects the heavy nuclei experiment negatively. The magnet in a sun synchronous can be shielded by the solar panels, which will allow the outside surface of the magnet cryostat to operate at a temperature as low as $230 \mathrm{~K}$. For a given amount of super fluid helium in the cryostat, the dewar life is increased about 30 percent by reducing the dewar vacuum shell temperature to $230 \mathrm{~K}$. As with the magnet system launched by an Atlas IIa, dipole moment correction coils are required.

Recent studies have centered on a Delta II launched version of ASTROMAG which is put into an earth trailing solar orbit. Under this scenario, the satellite is well outside of the earth's magnetic field (about 1.5 to 2.0 million kilometers from earth). The potential advantages of this kind of an arrangement are: 1) Large number of low energy particles are available for analysis. 2) Observation time is maximized. The life of the experiment can be extended out to beyond 3 years so the loss of collection area due to the downsizing of the experiment can be compensated for in part by increase mission time. 3) The magnet can be shielded from the sun by the solar panels. As a result of shielding the magnet and being well away from the earth, the surface temperature of the magnet cryostat can be as low as $120 \mathrm{~K}$. which has the effect of increasing the dewar life time as much as a factor of 1.4 (as compared to a tank with a vacuum shell at $230 \mathrm{~K}$ ) for a given amount of super fluid helium in the storage tank. 4) The net magnetic moment requirements are greatly relaxed for a magnet which is located well away from the earth. A single coil design may be reasonable provided the axis of 
the solenoid is perpendicular to a line between the satellite and the sun. A single coil magnet will have more bending power than a two coil solenoid system, because all of the coil mass can be concentrated in the single coil instead of split between two coils. Even if a two coil zero dipole magnet is required, the superconducting dipole moment correction coils are not needed. The primary disadvantage of this type of orbit is that the satellite mass is limited to 1025 to $1290 \mathrm{~kg}$ depending on the version of the Delta This has a negative effect on the size and the mass of the physics detectors and an effect on the bending power of the superconducting magnet. By necessity, only a single ended experiment can be launched into a solar orbit. The mass of the superconducting magnet, its cryostat and the helium would be in the 350 to $500 \mathrm{~kg}$ range.

\section{THE DELTA LAUNCHED SUPERCONDUCTING MAGNET SYSTEMS}

Table 1 compares the parameters of the space station version of ASTROMAG with the Atlas Ila version and the two Delta versions. The nominal dewar life for the space station version, the Atlas version and the low earth orbit versions of ASTROMAG are nominally 3.0 tc 3.5 years. The sun orbit Delta version will have a nominal dewar life of 4.2 to 4.8 years because the outer shell temperature is $120 \mathrm{~K}$ over much of the outer shell. (The portion of the outer shell which is next to the particle detectors will have a temperature of about $240 \mathrm{~K}$.) Studies of hybrid super fluid helium and solid hydrogen $\mathrm{cr}$, genic system for the deep space version show that there is the potential to increase the helium life time out to 6.0 io 6.5 years for a superconducting magnet system with the same overall mass of $500 \mathrm{~kg}$. Solid hydrogen hybrid systems have even greater potential when the cryostat vacuum shell temperature is closer to room temperature 9 . A hybrid cryogenic system is more complex and there are additional safety problems associated with it. (A cryogenic system which uses solid hydrogen for much of the cooling can only be launched by an expendable launch vehicle.)

Table 1

A comparison of Two Delta Magnet Versions and the Atlas Magnet Version with the Baseline Space Station Magnet for ASTROMAG

\begin{tabular}{|c|c|c|c|c|}
\hline & $\begin{array}{l}\text { Baseline } \\
\text { Space Station } \\
\text { Version } \\
\text { Low Orbh } \\
\end{array}$ & $\begin{array}{l}\text { Atlas } \\
\text { Free-flyer } \\
\text { Version } \\
\text { Low Orbit } \\
\end{array}$ & $\begin{array}{l}\text { Delta } \\
\text { Free-flyer } \\
\text { Version } \\
\text { Low Orblt } \\
\end{array}$ & $\begin{array}{l}\text { Delta } \\
\text { Free-flyer } \\
\text { Version } \\
\text { Sun Orbit } \\
\end{array}$ \\
\hline $\begin{array}{l}\text { Cryostat OD (m) } \\
\text { Cryostat Length (m) } \\
\text { Helium Tank Volume (liters) } \\
\text { Coil OD (m) } \\
\text { Coil Length (m) } \\
\text { Coil Thickness (m) } \\
\text { Coil Separation (m) } \\
\text { Number of Tui..ns per Coil } \\
\text { Design Current (A) } \\
\text { Magnet Stored Energy (MJ) } \\
\text { Physics Factor \#\# }\end{array}$ & $\begin{array}{r}2.120 \\
2.608 \\
3440 \\
1.660 \\
0.184 \\
0.050 \\
2.000 \\
2440 \\
810 \\
11.0 \\
0.987\end{array}$ & $\begin{array}{r}1.820 \\
2.350 \\
2250 \\
1.440 \\
0.220 \\
0.058 \\
1.670 \\
2928 \\
800 \\
11.6 \\
1.000\end{array}$ & $\begin{array}{r}1.550 \\
2.000 \\
1380 \\
1.232 \\
0.178 \\
0.052 \\
1.400 \\
2028 \\
925 \\
6.4 \\
0.700\end{array}$ & $\begin{array}{r}1.300 \\
1.650 \\
760 \\
1.050 \\
0.226 \\
0.052 \\
2544 \\
900 \\
3.6 \\
0.712\end{array}$ \\
\hline $\begin{array}{l}\text { Coil Mass (kg) } \\
\text { Switch \& Corrector Mass (kg) } \\
\text { Helium Mass (kg) } \\
\text { Dewar Mass (kg) } \\
\text { Charging System Mass \# (kg) }\end{array}$ & $\begin{array}{c}661 \\
30^{*} \\
500 \\
935 \\
150^{* *}\end{array}$ & $\begin{array}{r}414 \\
22 \\
327 \\
724 \\
104\end{array}$ & $\begin{array}{r}254 \\
13 \\
203 \\
470 \\
\sim 65\end{array}$ & $\begin{array}{r}134 \\
5 \\
110 \\
230 \\
-25\end{array}$ \\
\hline $\begin{array}{c}\text { Total Magnet Mass }(\mathrm{kg}) \\
\text { Magnet Weight Factor\# } \\
\text { Overall Mass }(\mathrm{kg})\end{array}$ & $\begin{array}{r}2276 \\
1.431 \\
-8500\end{array}$ & $\begin{array}{r}1591 \\
1.000 \\
5257\end{array}$ & $\begin{array}{r}1005 \\
0.632 \\
\sim 3000\end{array}$ & $\begin{array}{r}504 \\
0.317 \\
-1150\end{array}$ \\
\hline
\end{tabular}


The superconducting magnet coils will be made from a superconductor with insulated matrix dimensions of 1.55 by $2.10 \mathrm{~mm}$. (The projected insulation thickness will be $0.05 \mathrm{~mm}$ ) The primary matrix material is very pure aluminum with a RRR greater than 1000 at zero field. The ratio of aluminum to superconductor will be 3 to 1 . The conductor also has 1 part copper for each part of niobium titanium. The copper based conductor (1 to $1 \mathrm{Cu}$ to $\mathrm{Nb}$-Ti ratio) will be co-extruded with the pure aluminum matrix. The copper based conductor is a typical multifilamentary superconductor with filament diameters which are about 25 micrometers in diameter. In both Delta launched versions of ASTROMAG, the projected magnet operating current will be about 85 percent of the superconductor critical current at along the load line (at a temperature of $4.2 \mathrm{~K}$ ). The margin is even greater when the magnet operates at its design temperature of $1.8 \mathrm{~K}$.

Figure 1 shows the coil cross-sections for both the low earth orbit version and the solar orbit versions of the ASTROMAG magnet to be launched by a Delta launch vehicle. The table below each coil cross-section describes the parameters of the magnet coil above it. The coil mass given in the tables which are a part of Figure 1, is the mass for a single coil. The low earth orbit version has two such coils whereas the solar orbit version is shown has one coil.

Figure 2 shows the low earth orbit version of a Delta launched ASTROMAG magnet. Figure 3 shows the solar orbit version. The magnet shown in Figure 2 is capable of supplying a magnetic field to detectors at each er.j of the cryostat. The experimental packages are not shown in Figure 2. Figure 3 shows how a single detector package can be integrated with a single magnet coil and its cryostat in the solar orbit version. The detector operates in a carbon dioxide atmosphere at a pressure of $0.1013 \mathrm{MPa}(1 \mathrm{~atm})$; the interior of the magnet cryostat is in vacuum. The temperature within the experimental package will be about $240 \mathrm{~K}$. It is desirable for the rest of the magnet cryostat vacuum shell to be at 120 to $130 \mathrm{~K}$. An insulated section of the vacuum shell would separate the two regions.

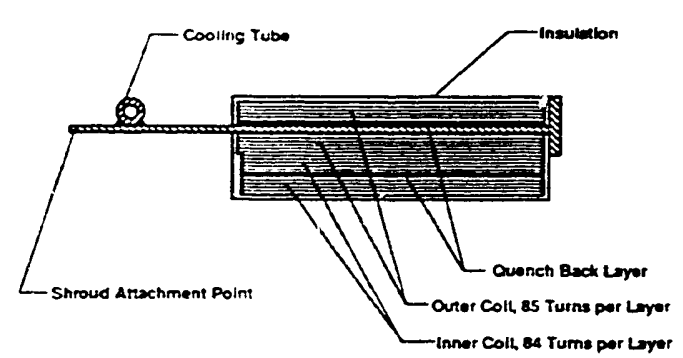

FREE-FLYER ALUMINUM MAGNET PARAMETERS

\begin{tabular}{|c|c|}
\hline 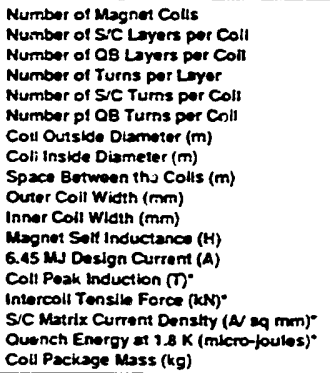 & $\begin{array}{r}22 \\
24 \\
2 \\
200085 \\
2028 \\
169 \\
1232 \\
7.128 \\
1.400 \\
178.50 \\
176.40 \\
1500 \\
925.0 \\
6.63 \\
2354 \\
325 \\
200 \\
127.2\end{array}$ \\
\hline
\end{tabular}

a) The Low Earth Orbit Version

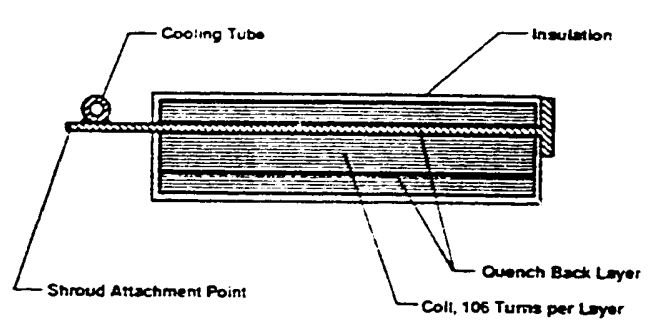

FREE-FL YER ALUMINUM MAGNET PARAMETERS

\begin{tabular}{|c|c|}
\hline 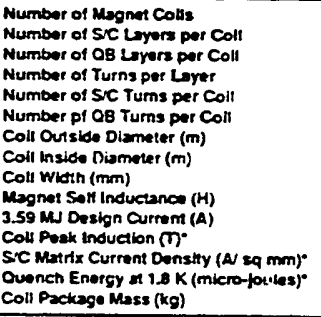 & $\begin{array}{r}22 \\
24 \\
2 \\
106 \\
254 \\
212 \\
1.050 \\
0.946 \\
2226 \\
8266 \\
6.00 \\
6.5 \\
316 \\
400 \\
134.2\end{array}$ \\
\hline
\end{tabular}

Figure 1 Coil Cross-sections for Delta Launched Versions of ASTROMAG 

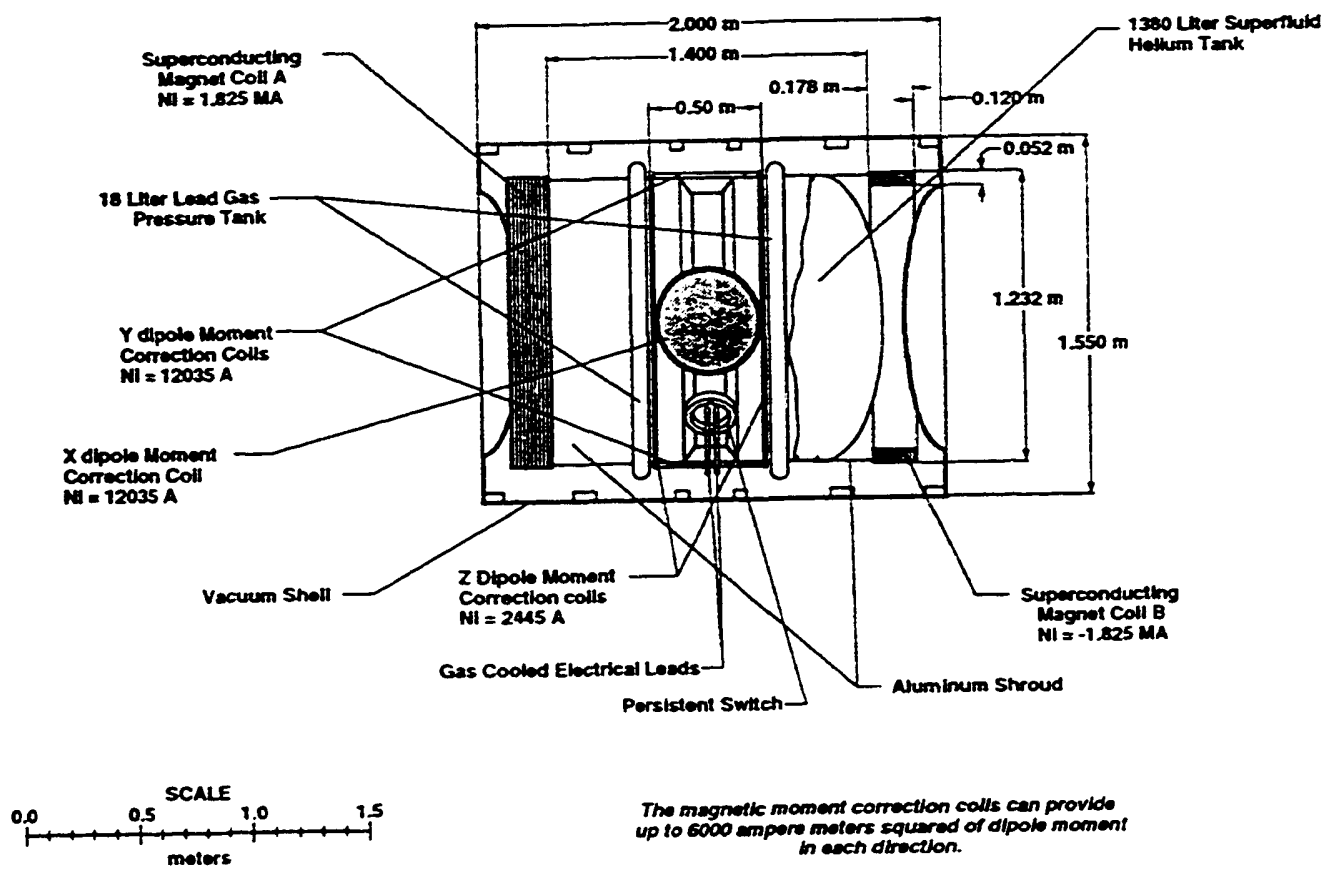
Tho magnetk moment correction colls can provide
up 106000 empore moters squered of olpoli moment
in ecch diruction.

Figure 2 A Low Earth Orbit Delta Launched Version of the ASTROMAG Magnet

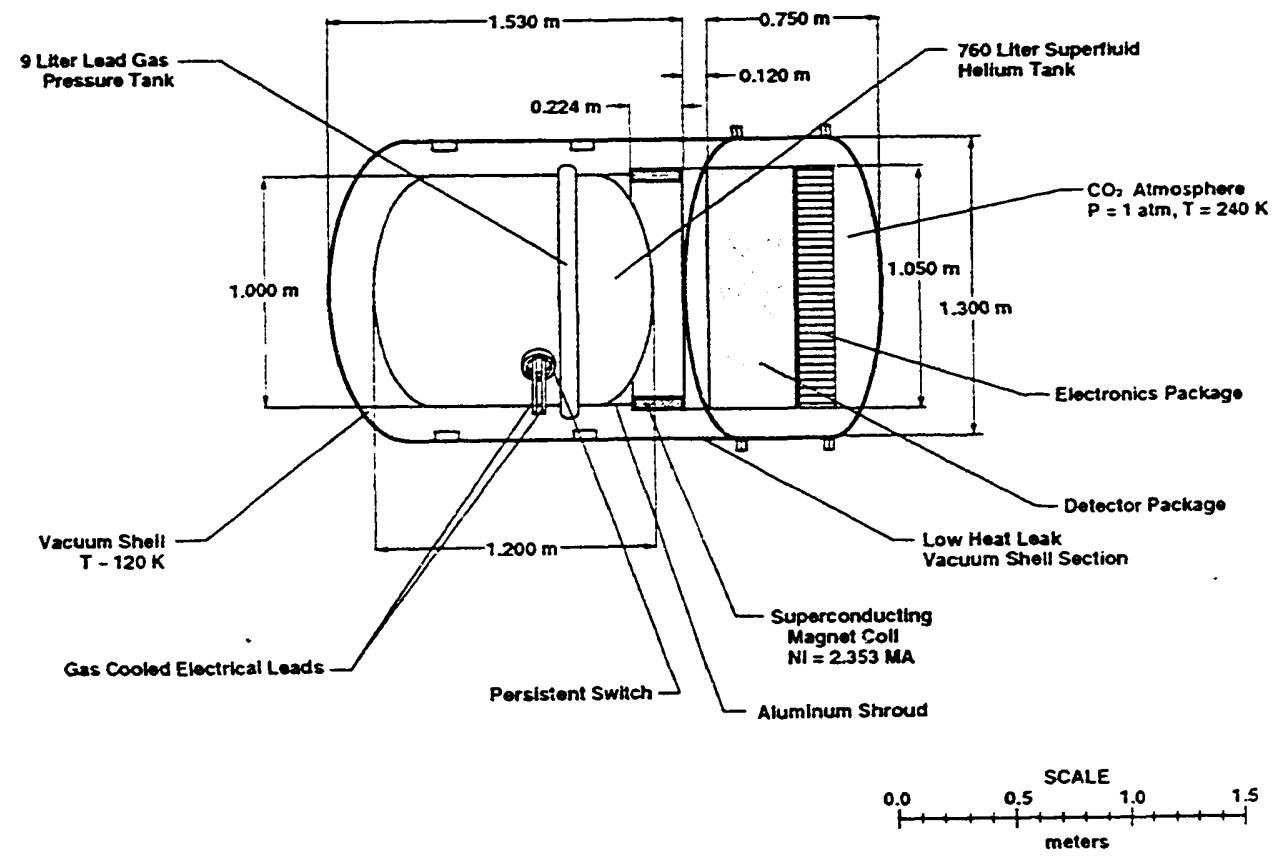

Figure 3 A Solar Orbit Delta Launched Version of the ASTROMAG Magnet 


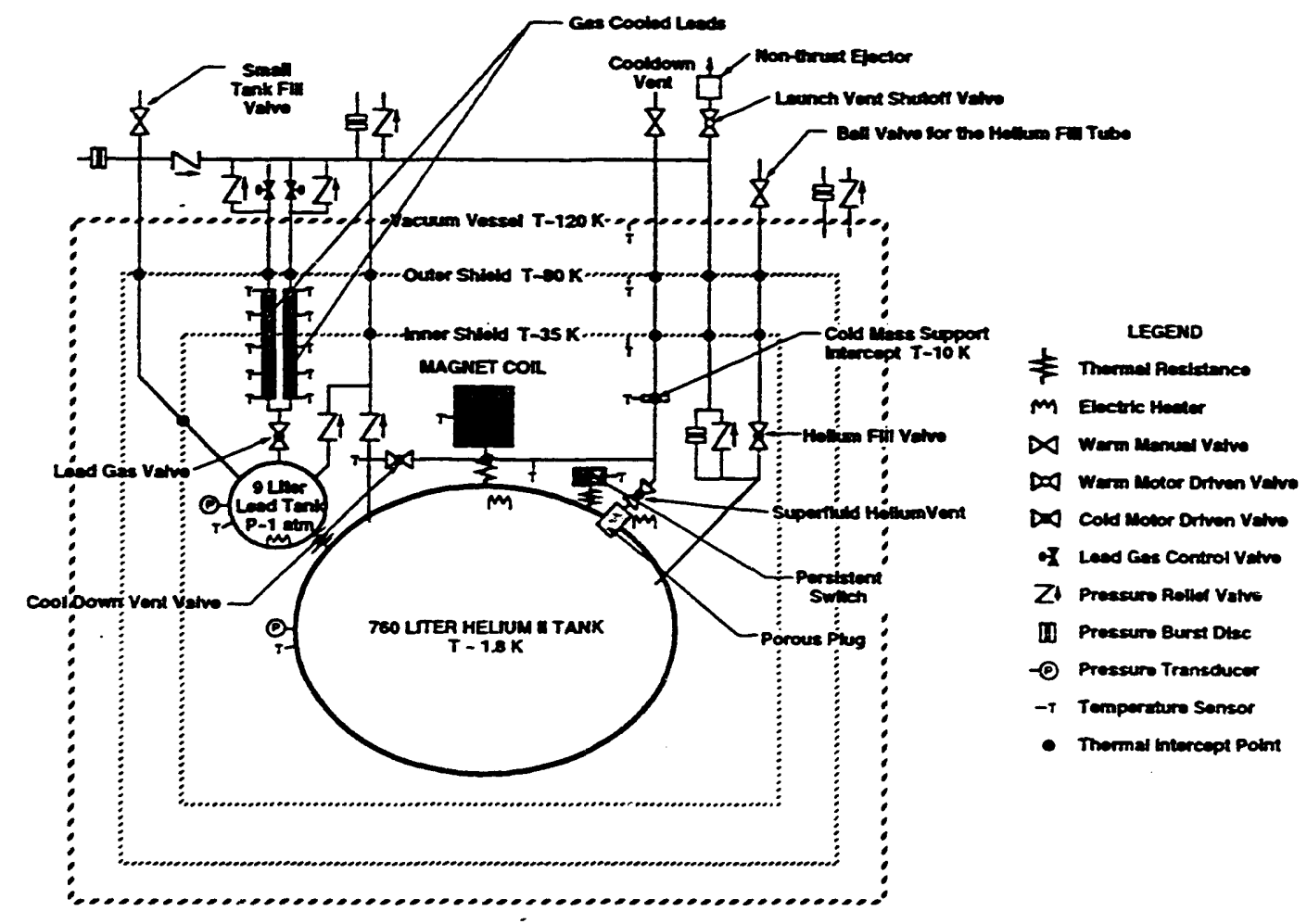

Figure 4 The $1.8 \mathrm{~K}$ Cooling System for the Solar Orbit Version of the ASTROMAG Magnet

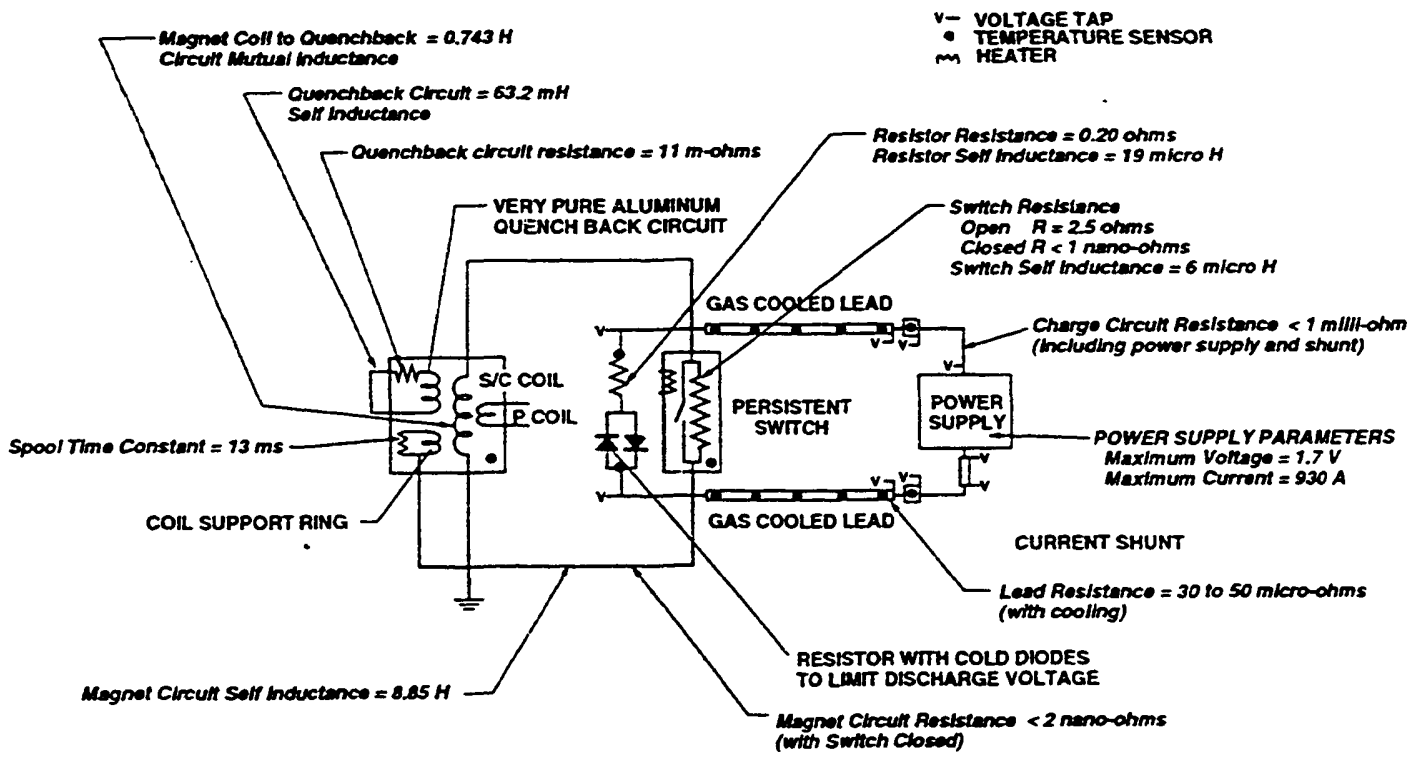

Figure 5 An Eiectrical Circuit Diagram for the Solar Orbit Version of a Delta Launched of the ASTROMAG Superconducting Magnet 
Figure 4 shows the super fluid helium cooling system for the solar orbit version of a Delta launched ASTROMAG. The cryostat vacuum shell, which is in the shadow of the solar panels, is expected to be at 120 to $130 \mathrm{~K}$ (except the end where the detectors are located which is at $230 \mathrm{~K}$ ). Two shields are shown in Figure 4. These shields are cooled by the boil off from the helium dewar. The magnet coil and the persistent switch are cooled from the super fluid heiium tank by conduction. Gas needed to cool the retractable gas cooled leads which suppiy current to the magnet during charging at the beginning of the mission (The magnet is charged once and is kept charged for the rest of the mission.) is supplied from the 9 liter tank which is pressurized by heating the helium. The separate helium tank is filled under pressure as the main storage tank is cooled down from room temperature. The boil off from the $\mathbf{7 6 0}$ liter helium II tank passes through a porous plug super fluid helium phase separator.

Figure 5 shows the power supply, persistent switch and the single superconducting coil which produces the magnetic field for the solar orbit version of ASTROMAG. Figure 5 shows key values of inductance, resistance and voltage in the superconducting magnet electrical system. The charge rate for power supply system shown in Figure 5 can be optimized to minimize the total amount of helium boil off during a charge ${ }^{10}$. This optimization will be carried a step further to minimize the total mass of helium boiled off during a charge, the mass of solar panels and the mass of backup batteries.

\section{CONCLUSION}

ASTROMAG magnets suitable for launch by a Delta rocket can be designed and built for either an $800 \mathrm{~km}$ sun synchronous orbit or an earth trailing orbit around the sun. The magnets for both orbits can benefit from having the outer vacuum shell operate at a temperature below room temperature. The dewar life in both cases will be sufficiently long to allow the required particle astrophysics data to be taken. In both cases the cryogenic system is straight forward and similar to helium dewars which have already been put into orbit.

\section{ACKNOWLEDGMENTS}

This work was performed at the Lawrence Berkeley Laboratory with the support of the NASA Office of Astrophysics and the Director, Office of Energy Research, Office of High Energy and Nuclear Physics, High Energy Physics Division, U. S. Department of Energy under Contract No. DE-ACO3-76SF00098.

\section{REFERENCES}

1. M. A. Green, et al., IEEE Trans. MAG-23, No. 2, p 1240, (1987)

2. "ASTROMA.G, Phase A Study Final Report," Advanced Missions and Analysis Office, Code 402, NASA Goddard Space Flight Center, Greenbelt, MD 20771 ( Dec. 1989).

3. M. A. Green and S. Castles, Advances in Cryogenic Engineering 33, p 631, (1987)

4. "ASTROMAG, Free-flyer Study Report," Advanced Missions and Analysis Office, Code 402, NASA Goddard Space Flight Center, Greenbelt, MD 20771 (March: 1991)

5. M. A. Green and G. F. Smoot, Cryogenics 32 , No. 2, p 91, (1992)

6. M. A. Green, Cryogenics 32, ICEC Supplement, p 332, (1992).

7. M. A. Green, IEEE Trans. MAG-27, No. 2, p 2260 , (1991)

8. M. A. Green, Cryogenics 33 , No. 4, p 417, (1993).

9. M. A. Green, Cryogenics $\underline{29}$, No. 5, p 484, (1989).

10. M. A. Green, IEEE Trans. Appl. S/C 3, No. 1, p 582, (1993). 
